\title{
A clinical approach to the assessment of left ventricular diastolic function by Doppler echocardiography: update 2003
}

\author{
S R Ommen, R A Nishimura
}

Heart 2003;89(Suppl III):iii 18-iii23

$\mathrm{P}$ atients with heart disease frequently have abnormalities of systolic function. However, it is now well recognised that abnormalities of diastolic function play a major role in producing the signs and symptoms of heart failure. ${ }^{12}$ Thirty to fifty per cent of patients with heart failure have normal systolic function, implicating diastolic dysfunction as a major pathophysiologic abnormality. It is important to understand and recognise abnormalities of diastolic filling of the heart for proper diagnosis, prognosis, and institution of treatment. ${ }^{3}$ Doppler echocardiography has become the primary tool for the assessment of diastolic function and left ventricular filling pressures. The purpose of this review is to summarise the physiology of diastole and develop a practical clinical approach for the non-invasive assessment of diastolic filling of the heart.

\section{DEFINITION OF DIASTOLE AND DIASTOLIC DYSFUNCTION \\ Cellular definition}

At the cellular level diastole can be considered as beginning when ATP hydrolyses and actin-myosin crossbridges become unlinked allowing for sarcomeric relaxation. This is integrally related to decreasing intracellular concentrations of calcium owing to enhanced sarcoplasmic reuptake of calcium. These cellular processes actually occur in some cells while other cells are still demonstrating active contraction. Thus cellular diastole may actually begin while left ventricular pressure is still rising.

Dysfunction at the cellular level is mediated principally via decreased ATP hydrolysis and/or impaired uptake of the intracellular calcium. When the actin-myosin interaction is prolonged, there is a delay and prolongation of sarcomeric expansion. Additionally, in the case of regional ischaemia, there may be regional cellular impairment such that the heart ceases to function as a syncytium.

\section{Mechanical definition}

Mechanically, diastole is considered to begin when the pressure within the left ventricle begins to fall-that is, during the isovolumic relaxation phase. This would occur after a significant number of myocardial cells had entered cellular diastole and is a metabolically active phase. The left ventricular pressure will continue to fall rapidly, with opening of the mitral valve occurring when the left ventricular pressure falls below the left atrial pressure. In the normal heart there is a suction effect after mitral valve opening that causes rapid early filling. Left ventricular relaxation will normally end in the first third of filling so that the remainder of filling is dependent upon the passive tissue properties as well as contributions from ventricular interaction, pericardial restraint, and the viscoelastic forces of the myocardium. At the end of diastole, atrial contraction will restore the full preload of the ventricle before the onset of contraction.
The processes that impair diastole at the cellular level also alter active mechanical relaxation and subsequent filling. Additionally, increased myocardial fibrosis or infiltrative disease can increase the stiffness of the heart during passive filling of the left ventricle. Enhanced ventricular interaction or pericardial restraint may also impact on passive filling. The end result of impairment of either relaxation or filling is that intracardiac pressures need to increase inappropriately to achieve adequate left ventricular filling volumes for the next systolic contraction.

\section{Clinical definition}

For the practising physician it is difficult to understand or measure in isolation the contribution of the multiple interrelated components of the cellular and mechanical mechanisms comprising diastole. ${ }^{4}$ From a simplistic standpoint, clinical diastole is the process or phase where the heart, as a global operating chamber, relaxes and fills with blood in preparation for the next contraction. The simplified, albeit still interrelated sequence of events is: (1) relaxation, (2) suction, (3) filling, and (4) atrial contraction (fig 1). In this scheme, diastolic dysfunction is any abnormality that causes impaired relaxation (and decreased ventricular suction), poor filling, or loss of atrial contraction. One or more of these abnormalities results in increased pressures to achieve an adequate filling volume. This translates into "diastolic dysfunction" and results in the signs and symptoms of heart failure.

\section{PROGRESSION OF DIASTOLIC DYSFUNCTION AND RELATION TO DOPPLER MITRAL FLOW CURVES}

There is a progression of diastolic dysfunction that has been described for different disease states (fig 2). Understanding the relation between various Doppler velocity curves and left ventricular filling abnormalities has allowed a non-invasive approach to identify the severity of diastolic dysfunction in patients. Different grades of diastolic dysfunction (I, II, III, IV) can be determined, and these Doppler velocity curves can be used for diagnosis, prognosis, and determination of therapy in patients with suspected diastolic dysfunction.

Left ventricular diastole is characterised in the normal state by ventricular relaxation and a rapid fall in left ventricular pressure. Continued relaxation involves the ATP dependent uptake of intracellular calcium into the sarcoplasmic reticulum. Elastic recoil and suction then occur after mitral valve opening and result in rapid flow across the mitral valve. Left ventricular compliance (distensibility) is higher than atrial compliance during diastole and further favours filling of the ventricle (that is, the atrium acts as a conduit). This results in rapid myocardial relaxation velocity and rapid, unimpeded flow across the valve. These changes in the relation of left ventricular and left atrial pressures are reflected in the mitral valve inflow velocities, derived from Doppler echocardiography. There is a rapid velocity early inflow that occurs with mitral valve opening (E wave) followed by deceleration of 


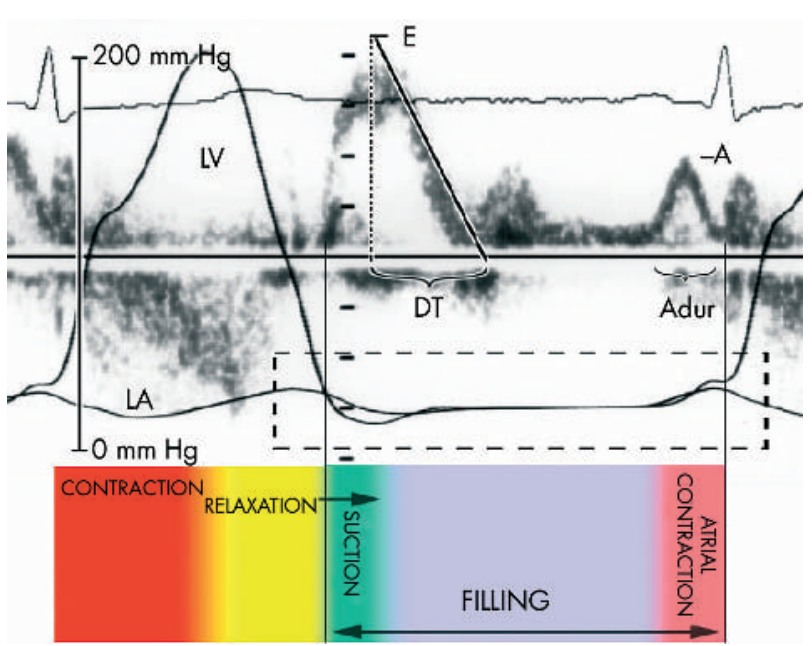

Figure 1 The phases of left ventricular filling are relaxation, suction, filling, and atrial contraction as depicted in these simultaneous invasive pressure curves and Doppler echocardiography. A, mitral filling at atrial contraction; Adur, duration of mitral A wave; DT, mitral deceleration time; $E$, mitral early filling wave; LA, left atrial pressure curve; LV, left ventricular pressure curve.

flow as left ventricular pressure rises to meet left atrial pressure. This may be followed by a brief period where there is no pressure gradient across the valve (diastasis). At end diastole, with the contraction of the atrium there is a second wave (A wave) of blood flow into the ventricle.

The initial abnormality of diastolic function is loss of the elastic recoil/suction forces in early diastole. This and subsequent slowing of the energy requiring myocardial relaxation can be identified by a decreased left atrial-left ventricular (LA-LV) pressure gradient and increased reliance on atrial contribution to left ventricular filling. In this state, the mitral E velocity is decreased and flow into the ventricle continues throughout diastole. Because of incomplete atrial emptying (and therefore increased atrial preload), there is an increase in the velocity and importance of the A wave. The Doppler velocity curve of a low E/A ratio and prolongation of deceleration time represents grade I diastolic dysfunction (abnormal relaxation pattern). These patients may have

Progression of diastolic dysfunction
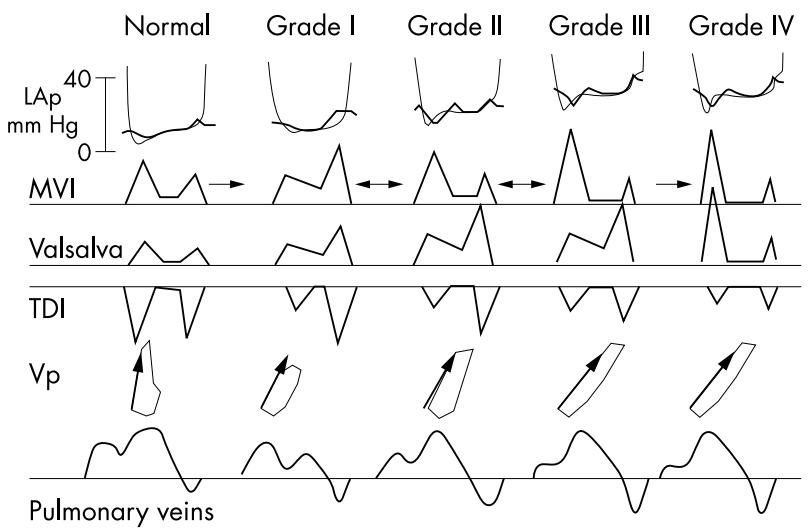

Figure 2 The progression of left ventricular diastolic dysfunction can be readily assessed using a combination of Doppler echocardiographic variables. Each successive grade represents a worsening state of diastolic dysfunction. LAp, left atrial pressure; $M V I$, mitral valve inflow; TDI, tissue Doppler imaging; Valsalva, response of mitral valve inflow to Valsalva manoeuvre; $\mathrm{Vp}$, mitral inflow propagation velocity.

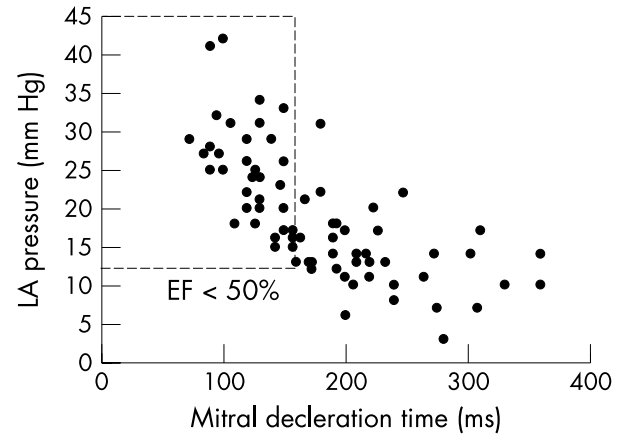

Figure 3 Relation between mitral deceleration time and left atrial pressure as assessed from three separate simultaneous Doppler catheterisation studies. EF, left ventricular ejection fraction; LA, left atrial. Adapted from Nishimura et al, ${ }^{6}$, Ommen et al, ${ }^{7}$ and Yamamoto et al. ${ }^{13}$

normal or only mildly increased filling pressures at rest but may develop symptomatic increases in filling pressures with exercise as the diastolic filling time shortens.

As disease progresses, there is further decline of active myocardial relaxation and the onset of operative compliance abnormalities. Filling of the left ventricle becomes increasingly dependent on the LA-LV pressure gradient (driving force) which can be maintained only by increasing left atrial pressure. In this state, left atrial pressure is raised throughout the cardiac cycle with a transient decrease as the atrium empties in ventricular diastole. Increased mean left atrial pressure "pseudonormalises" the flow from the left atrium to left ventricle with an increase in the early inflow velocity and shortening of the deceleration time. This state of diastole is characterised by mitral inflow signals that are similar to that of the normal state: rapid early inflow (increased $\mathrm{E}$ wave) and less prominent flow at atrial contraction (A wave). This is grade II diastolic dysfunction (pseudonormal pattern).

In the latter stages of diastolic deterioration, left ventricular chamber compliance is severely impaired such that there is a larger increase in pressure for small increases in ventricular volume. Here the early mitral inflow is very rapid (high E wave) with rapid equilibration of left atrial and left ventricular pressure that truncates the $\mathrm{E}$ wave (very short deceleration time). The pressures and operating compliance are such that there is very little contribution to filling the ventricle from atrial contraction (small A wave). This is the restrictive pattern, representing grade III-IV of diastolic dysfunction. An individual patient may progress or regress between grades I, II, and III diastolic dysfunction depending upon the state of compensation in filling pressures at the time of the echocardiographic examination. However, in

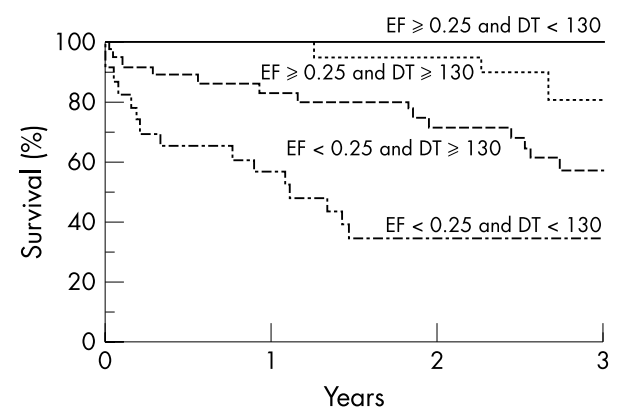

Figure 4 Relation between systolic and diastolic function and overall survival highlights the critical role of diastolic function for the prognosis of patients with impaired left ventricular systolic function. DT, Deceleration time; EF, ejection fraction. Reproduced from Rihal et al, ${ }^{8}$ with permission. 

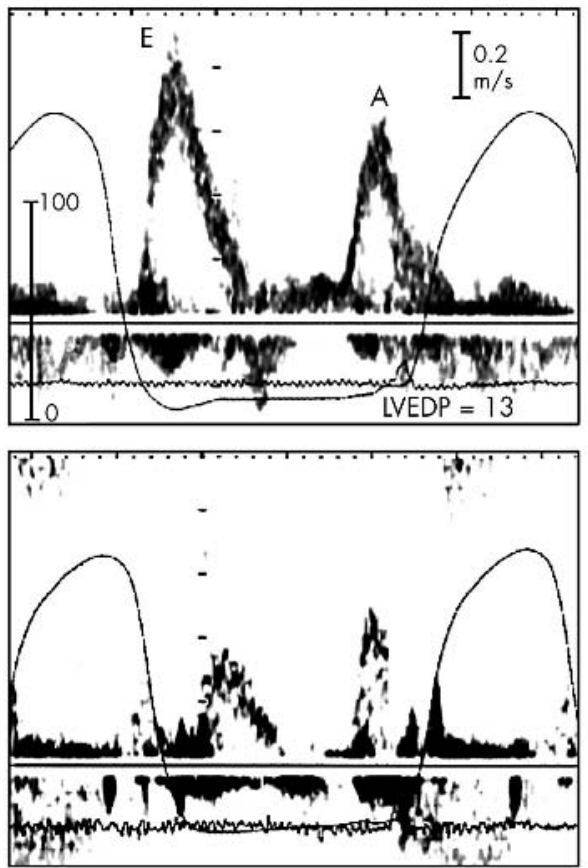

Normal
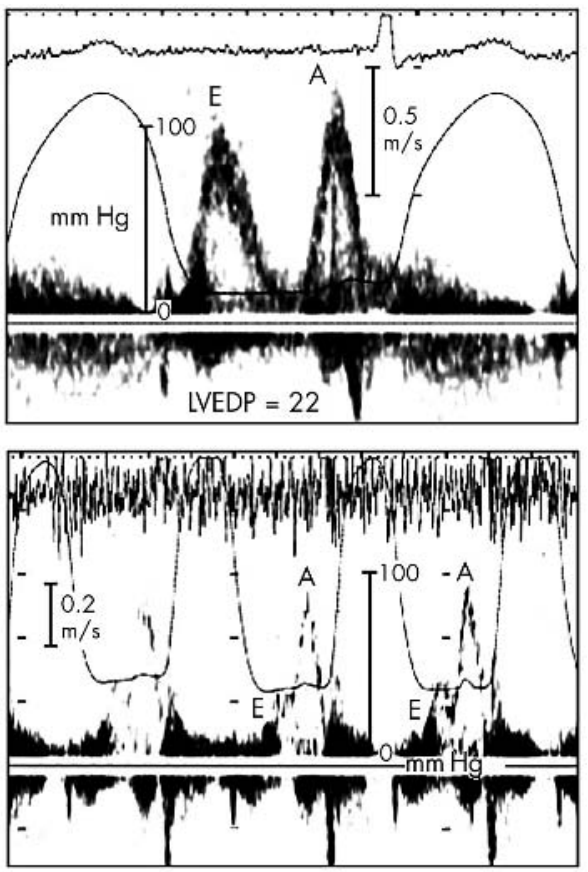

Abnormal
Figure 5 The response of the mitral inflow to preload manipulation can be useful in predicting filling pressures. On the left is a normal response to the Valsalva manoeuvre with proportional decreases in both mitral $E$ and $A$ waves in a patient with normal filling pressures. On the right, the Valsalva manoeuvre results in disproportionate decrease in mitral $E$ wave in a patient with raised filling pressure. A decrease in the mitral $E / A$ ratio of 0.5 or more is a highly specific indicator of increased filling pressures. A, mitral filling at atrial contraction; $E$, mitral early filling wave; LVEDP, left ventricular end diastolic pressure; $\mathrm{m} / \mathrm{s}$, metres per second. severe end stage disease, the restrictive pattern persists despite manipulation of filling pressures and may be irreversible (grade IV).

The change in the mitral flow velocity patterns with advancing diastolic dysfunction has been described particularly in
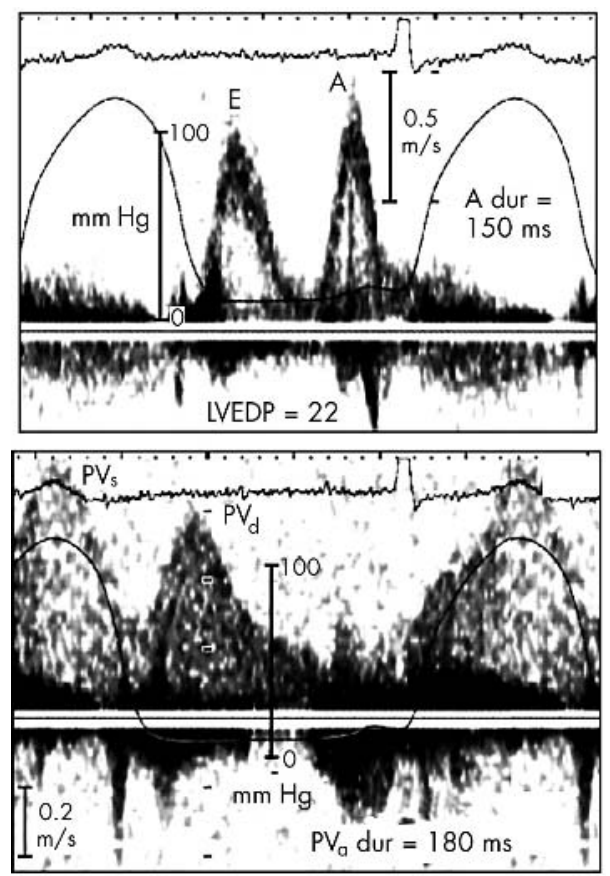

Figure 6 The duration of flow at atrial contraction into the left ventricle as compared to duration of flow reversal into the pulmonary veins can indicate end diastolic filling pressure. In this example, flow duration is much longer on the pulmonary vein Doppler signal ( $180 \mathrm{~ms} v 150 \mathrm{~ms}$ ) indicating raised left ventricular end diastolic pressure. A, mitral filling at atrial contraction; $\mathrm{E}$, mitral early filling wave; LVEDP, left ventricular end diastolic pressure; $P V_{a}$ dur, pulmonary vein atrial reversal duration; $P V_{d}$, pulmonary vein diastolic forward flow, $\mathrm{PV}_{\mathrm{s}}$, pulmonary vein systolic forward flow. those with concomitant systolic dysfunction. ${ }^{45}$ In this group of patients there is a strong correlation between mitral inflow parameters and filling pressures allowing prediction of elevated filling pressures with high specificity (fig 3). ${ }^{67}$ Furthermore, worsening mitral inflow patterns have been correlated with worsening prognosis in several sets of patients (fig 4$).^{8-12}$

\section{COMPREHENSIVE DOPPLER EXAMINATION}

Although the mitral inflow velocity curves are predictive of filling pressures and can determine prognosis in subgroups of patients, there are major pitfalls that can limit the utility of mitral inflow in the general assessment of diastolic function. First, prediction of left ventricular filling pressures by mitral valve inflow velocities appears to be accurate only in those patients with systolic dysfunction, as patients with normal systolic function show wide scatter of filling pressures. ${ }^{413}$ This is related to the progression of disease that occurs, so that in an advanced state of diastolic dysfunction the mitral inflow pattern looks identical to the normal state. The reason for the confusing similarity is that normal hearts have rapid relaxation and fall in left ventricular pressure such that blood is "sucked" across the mitral valve. Those with grade II diastolic dysfunction display a "pseudonormal" mitral inflow from the raised left atrial pressure where blood is "pushed" across the valve. There also are data that demonstrate that patients with an E/A ratio $<1$ can have raised left ventricular filling pressures as they transition to higher degrees of diastolic abnormality. ${ }^{14}{ }^{15}$ The distinction of normal versus pseudonormal filling is difficult using the mitral inflow alone and in most patients other parameters will be necessary to complete the assessment.

\section{Preload alteration}

Reducing left ventricular preload, using glyceryl trinitrate or the Valsalva manoeuvre, has been used as a means of unmasking patients with elevated filling pressures. Those patients with a normal pattern and filling pressure will simply show a hypovolaemic response (reduction in all 


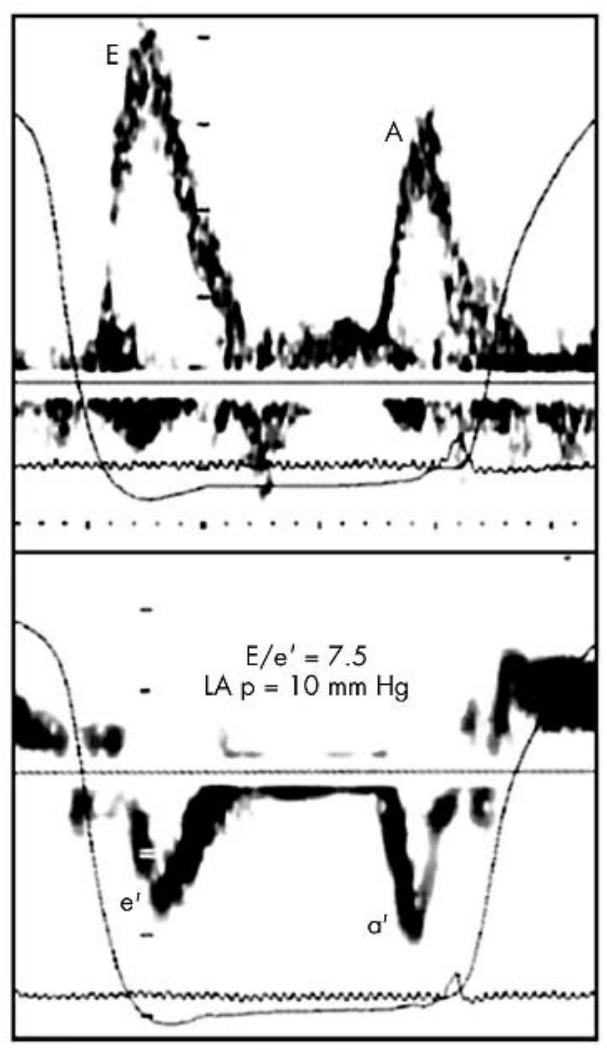

Normal

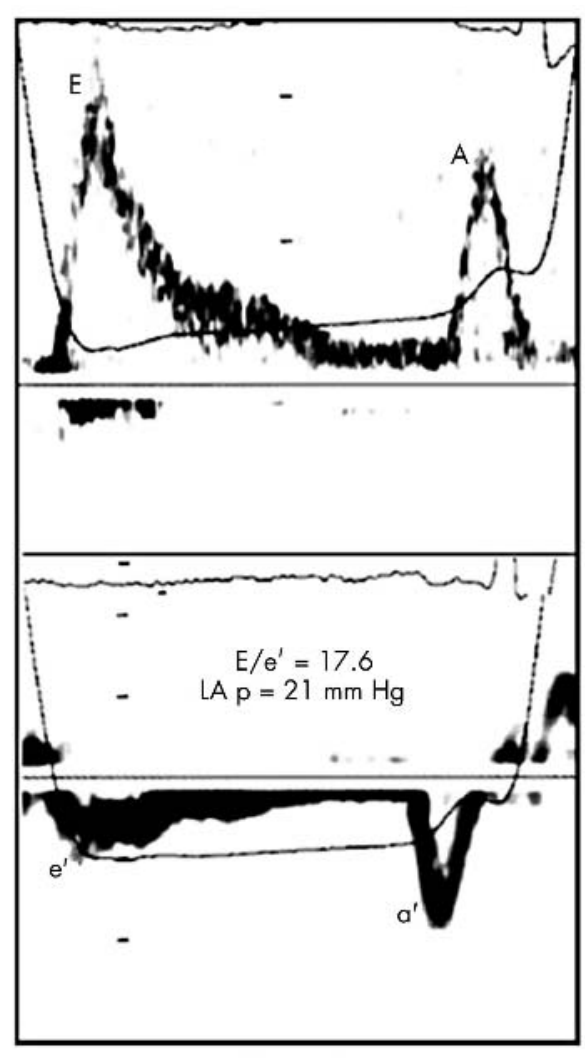

Abnormal
Figure 7 The tissue Doppler velocity of the mitral annulus can help distinguish between normal and abnormal filling. These examples are from two patients with normal systolic function and similar mitral inflow signals. On the left, a normal mitral annular velocity and $\mathrm{E} / \mathrm{e}^{\prime}$ ratio indicate normal filling pressures. On the right, the mitral annular $\mathrm{e}^{\prime}$ velocity is reduced and the $\mathrm{E} / \mathrm{e}^{\prime}$ ratio elevated in a patient with high left atrial pressure. A, mitral filling at atrial contraction; $a^{\prime}$, late mitral annulus diastolic velocity; $E$, mitral early filling wave; $e^{\prime}$, early mitral annulus diastolic velocity; $E / e^{\prime}$, ratio of mitral early filling wave to early mitral annulus velocity;

LA $p$, left atrial pressure. velocities) (fig 5). The reduction of preload (left atrial pressure) in patients with "pseudonormal" flow (raised filling pressures coupled with abnormal relaxation) should reveal the underlying abnormal relaxation pattern. ${ }^{74-17}$ Reported data suggest that patients who can reduce the E/A ratio by an absolute value of 0.5 (that is, from 1.3 to 0.8 ) or more have raised filling pressures, independent of systolic function. Unfortunately, preload alteration is not universally practical in clinical practice. Some patients are not able to perform an adequate Valsalva manoeuvre and careful attention to signal acquisition and measurement is crucial. In a recent series adequate signals were obtained in only $61 \%$ of patients. ${ }^{7}$ The administration of nitrates to alter preload, while more predictable, is not feasible in many practice settings.

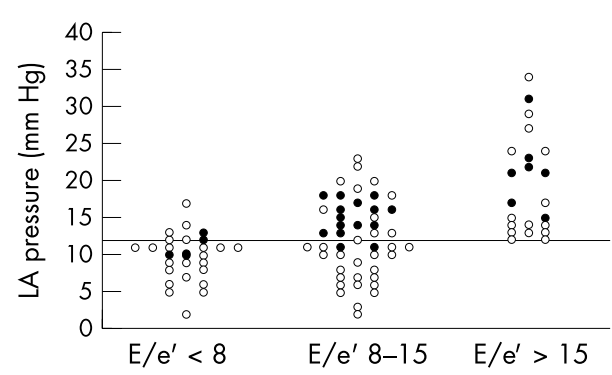

Figure 8 Cut-off values using the ratio of mitral early filling wave to early mitral annulus velocity $\left(\mathrm{E} / \mathrm{e}^{\prime}\right)$ can be used to group patients according to filling pressures. Those with high ratios (> 15) have high filling pressures, while those with very low ratios tend to have normal filling pressures. The intermediate group requires additional information to correctly classify diastolic function. The closed circles represent those patients with other Doppler parameters suggesting high filling pressures. Reproduced from Ommen et al, ${ }^{7}$ with permission.

\section{Pulmonary venous Doppler velocities}

Pulmonary venous flow is used as an adjunct to mitral inflow. Pulmonary venous flow pattern has also been well characterised and provides additive insight to diastolic filling of the left ventricle. ${ }^{18-24}$ The pattern of flow (systolic versus diastolic predominance) has been proposed as a predictor of left atrial pressure but is not applicable in unselected patients because of the multiple contributions to these velocities. ${ }^{7}$ Comparison of the duration of flow at atrial contraction across the mitral valve (on the mitral inflow velocity curve) and the duration of reversal flow back into the pulmonary veins (on the pulmonary venous velocity curves) has been repeatedly demonstrated to reflect the left ventricular end diastolic pressure. ${ }^{22}$ As filling pressures and therefore operating chamber characteristics worsen, a worsening relative compliance of the left ventricle compared with the pulmonary venous circuit is observed. Transmitral flow at atrial contraction is shortened while retrograde flow at atrial contraction into the low resistance pulmonary venous circuit continues for a longer duration. If the duration of atrial reversal flow in the pulmonary vein exceeds by more than $30 \mathrm{~ms}$ the duration of flow across the mitral valve, raised left ventricular end diastolic pressure can be diagnosed with high specificity (fig 6)..$^{71314}$ The major limitations to the use of the pulmonary venous signals are that these signals are difficult to obtain and interpret. The technical feasibility of obtaining adequate signals has been reported at less than $80 \%$ of unselected patients. ${ }^{7}$

\section{Tissue Doppler velocities}

The velocity of the mitral annulus, representing velocity of changes in left ventricular long axis dimensions, has been related to measures of systolic and diastolic left ventricular performance. ${ }^{25-30}$ The diastolic velocity has been proposed as representing the intrinsic speed of myocardial relaxation. 


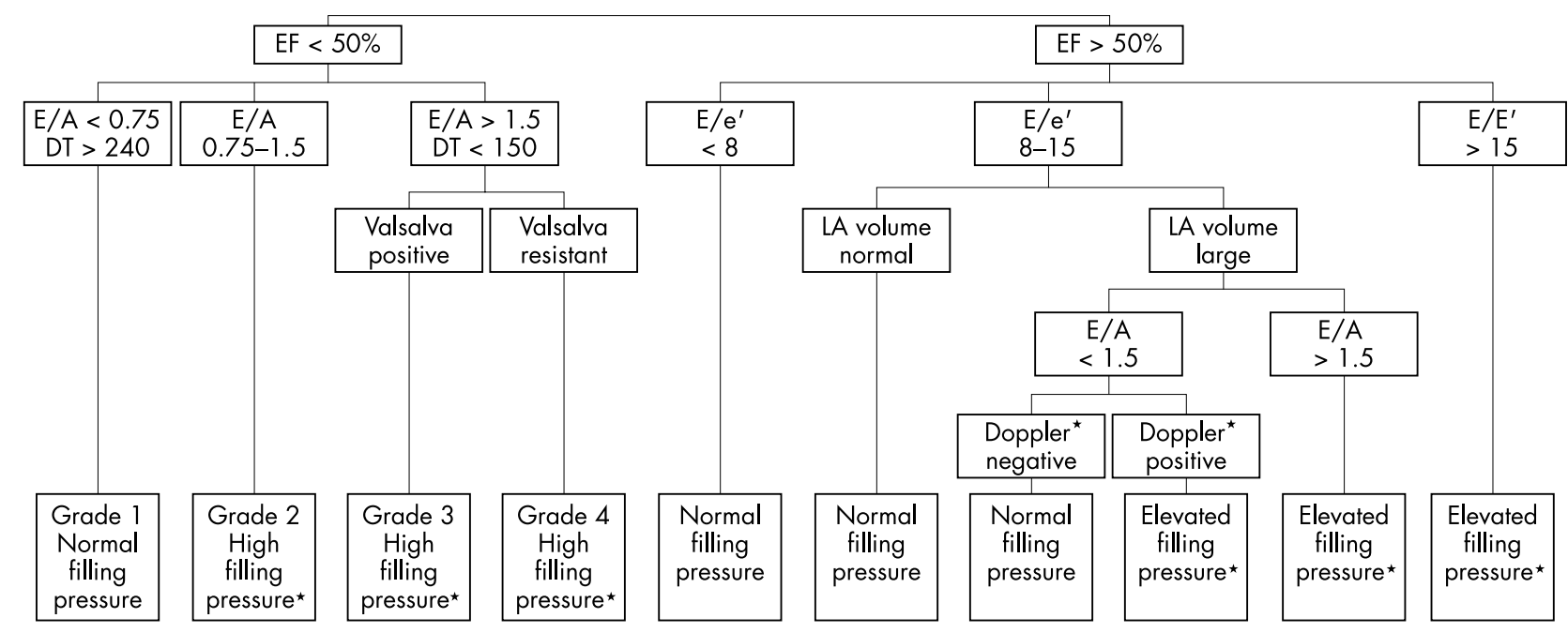

Figure 9 Screening assessment for diastolic function and filling pressures. Two dimensional and Doppler echocardiographic variables can be used to readily classify diastolic function. *In general, high filling pressures should be confirmed with multiple parameters (that is, E/e', E/Vp, A-dur difference, response to Valsalva manoeuvre, TR velocity, etc).

This may then be used to determine the difference between the effect of "suction" (normal E wave and rapid early mitral annular velocity) versus "pushing" with high left atrial pressure causing an increase in transmitral flow (normal E wave with reduced early mitral annular velocity).

The ventricle elongates in two distinct phases. The annular velocities recorded during these two phases are called $\mathrm{e}^{\prime}$ and $\mathrm{a}^{\prime}$, which correspond temporally with the mitral $\mathrm{E}$ and $\mathrm{A}$ waves. The velocity of $\mathrm{e}^{\prime}$ has been modestly correlated to the time constant of relaxation. A pattern of progression of diastolic dysfunction of the Doppler tissue velocities can be seen (fig 2). In the initial stages of diastolic dysfunction, the relaxation velocity $\left(\mathrm{e}^{\prime}\right)$ decreases and remains reduced throughout the remaining stages of impaired diastole. ${ }^{30}$

Subsequent investigations have shown that combining the mitral inflow with the mitral annular velocity into a ratio $\left(\mathrm{E} / \mathrm{e}^{\prime}\right)$ can predict left ventricular filling pressure (fig 7). ${ }^{71-33}$ The ability of this ratio to predict filling pressure has been demonstrated in patients with normal sinus rhythm, sinus tachycardia, preserved systolic function, atrial fibrillation, and in patients with hypertrophic cardiomyopathy. This combination may resolve the issue of discriminating normal from pseudonormal filling. Patients with diastolic dysfunction and a normal appearing mitral inflow pattern will also have a reduced mitral annular velocity $\left(\mathrm{e}^{\prime}\right)$ and an elevated $\mathrm{E} / \mathrm{e}^{\prime}$ ratio. Again, cut off values can easily be selected from published data. If $\mathrm{E} / \mathrm{e}^{\prime}$ is $>15$, left ventricular filling pressure is raised, and when E/e' is $<8$ filling pressure is low (fig 8 ). However, between $8-15$ there is considerable variability in filling pressure. ${ }^{7}$

\section{Flow propagation}

Assessment of flow propagation into the left ventricle is another technique that provides better ability to predict filling pressures. ${ }^{34-36}$ In the normal state, flow rapidly propagates into the left ventricular (fig 2). Early stage relaxation abnormalities show a blunting of flow propagation. The propagation velocity does not show a pseudonormalisation and therefore can be used in all levels of systolic function.

Similar to the tissue Doppler velocities, colour M mode flow propagation has been combined in a ratio with the mitral E velocity to provide an "adjusted" parameter (E/Vp) with strong correlation to filling pressures and prognosis. ${ }^{7234-37}$ The chief limitations of this tool are lack of consensus on technique and theoretical concerns that this will be invalid in small left ventricular cavities.

\section{APPLICATION TO CLINICAL PRACTICE}

These findings described herein can be incorporated into clinical practice to provide a non-invasive assessment of diastolic filling of the heart (fig 9). Doppler variables should always be viewed in context of ventricular size and function, as well as left atrial size. ${ }^{21}$ For example, patients with preserved systolic function and normal left atrial size will almost always have normal diastolic filling pressures and normal diastolic function. The mitral inflow and mitral annulus velocity signals can be obtained in over $90 \%$ of patients. Thus these two Doppler parameters, in conjunction with the two dimensional echocardiographic findings, represent the most efficient means of initial diastolic assessment. The diagnosis of diastolic dysfunction and raised filling pressures should always be considered in the context of the clinical presentation and other features (estimated pulmonary artery pressures, for example).

\section{Impaired systolic function}

For patients with reduced systolic function, diastolic function is abnormal and the mitral inflow parameters are predictive of filling pressure. If the mitral deceleration time is less than $150 \mathrm{~ms}$ and $\mathrm{E} / \mathrm{A}>1.5$ the filling pressures are raised. Among these patients, those who can reduce their E/A ratio by at least 0.5 can be called grade III diastolic dysfunction while those with an unchanged E/A ratio are grade IV. Patients with a low E/A ratio $(<0.75)$ and prolonged DT $(>240 \mathrm{~ms})$ can be said to have low to normal filling pressure and grade I diastolic dysfunction. Patients that fall in between are called grade II. The E/ $/ \mathrm{e}^{\prime}$ ratio can also be applied in this group as described above. Patients with grade I dysfunction should have a lower $\mathrm{E} / \mathrm{e}^{\prime}$ ratio, while grade II-IV will have a raised $\mathrm{E} / \mathrm{e}^{\prime}$ ratio. This stepwise approach facilitates classification of diastolic function and filling pressures in most patients.

\section{Preserved systolic function}

Patients with E/e' $<8$ can be classified as normal filling pressure and if there is a normal left atrial size, normal diastolic function can be diagnosed. Those with $\mathrm{E} / \mathrm{e}^{\prime}>15$ have raised filling pressure. In the intermediate group $\left(\mathrm{E} / \mathrm{e}^{\prime}\right.$ 8-15), abnormal filling pressure can be predicted only if other Doppler variables meet high specificity cut-off values. 
Therefore, a patient with intermediate E/e' can be classified as raised filling pressure if the E/A ratio decreases by more the 0.5 during the Valsalva manoeuvre, or the pulmonary venous A wave duration exceeds the mitral a wave duration by at least $30 \mathrm{~ms}$. If neither of these conditions is met and the left atrial size is normal, filling pressure is to be likely normal.

\section{CONCLUSION}

Diastolic abnormalities of the left ventricle result in significant morbidity and adverse outcomes. Assessment of true myocardial and cellular level diastole is complex and elusive; however, clinically meaningful information regarding chamber characteristics is available. The assessment of diastolic function and left ventricular filling pressures continues to evolve. Using a stepwise, evidence based approach involving readily obtained Doppler echocardiographic variables, left ventricular filling pressures and the stage of diastolic dysfunction can be obtained in most patients.

\section{Authors' affiliations}

S R Ommen, R A Nishimura, Division of Cardiovascular Diseases, Mayo Clinic, Rochester, Minnesota, USA

Correspondence to: Steve R Ommen, MD, Division of Cardiovascular Diseases, Mayo Clinic, 200 First Street SW, Rochester, MN 55906, USA; omen.steve@mayo.edu

\section{REFERENCES}

1 Senni M, Tribouilloy CM, Rodeheffer RJ, et al. Congestive heart failure in the community: a study of all incident cases in Olmsted County, Minnesota, in 1991. Circulation 1998:98:2282-9.

2 Redfield MM, Jacobsen SJ, Burnett JC Jr, et al. Burden of systolic and diastolic ventricular dysfunction in the community: appreciating the scope of the heart failure epidemic. JAMA 2003;289:194-202

3 Vasan R, Levy D. Defining diastolic heart failure: a call for standardized diagnostic criteria. Circulation 2000;101:2118-21.

4 Nishimura RA, Tajik AJ. Evaluation of diastolic filling of left ventricle in health and disease: Doppler echocardiography is the clinician's Rosetta Stone. J Am Coll Cardiol 1997;30:8-18.

5 Appleton C, Hatle L. The natural history of left ventricular filling abnormalities: assessment of two-dimensional and Doppler echocardiography. Echocardiography 1992;9:437-47

6 Nishimura RA, Appleton CP, Redfield MM, et al. Noninvasive doppler echocardiographic evaluation of left ventricular filling pressures in patients with cardiomyopathies: a simultaneous Doppler echocardiographic and cardiac catheterization study. J Am Coll Cardiol 1996;28:1226-33.

7 Ommen SR, Nishimura RA, Appleton CP, et al. Clinical utility of Doppler echocardiography and tissue Doppler imaging in the estimation of left ventricular filling pressures: a comparative simultaneous Dopplercatheterization study. Circulation 2000;102:1788-94

8 Rihal CS, Nishimura RA, Hatle LK, et al. Systolic and diastolic dysfunction in patients with clinical diagnosis of dilated cardiomyopathy. Relation to symptoms and prognosis. Circulation 1994;90:2772-9.

9 Temporelli PL, Corra U, Imparato A, et al. Reversible restrictive left ventricular diastolic filling with optimized oral therapy predicts a more favorable prognosis in patients with chronic heart failure. J Am Coll Cardiol 1998;31:1591-7

10 Vasan RS, Benjamin EJ, Levy D. Prevalence, clinical features and prognosis of diastolic heart failure: an epidemiologic perspective. J Am Coll Cardiol 1995;26:1565-74.

11 Xie GY, Berk MR, Smith MD, et al. Prognostic value of Doppler transmitral flow patterns in patients with congestive heart failure. J Am Coll Cardiol 1994:24:132-9.

12 Moller J, Sondergaard E, Seward J, et al. Ratio of left ventricular peak E-wave velocity to flow propagation velocity assessed by color M-mode Doppler echocardiography in first myocardial infarction: prognostic and clinical implications. J Am Coll Cardiol 2000;35:363-70.

13 Yamamoto K, Nishimura RA, Chaliki HP et al. Determination of leff ventricular filling pressure by Doppler echocardiography in patients with coronary artery disease: critical role of left ventricular systolic function. J Am Coll Cardiol 1997;30:1819-26.
14 Brunner-LaRocca H, Rickli $\mathrm{H}$, Attenhofer-Jost $\mathrm{C}$, et al. Left ventricular enddiastolic pressure can be estimated by either changes in transmitral inflow pattern during Valsalva maneuver or analysis of pulmonary venous flow. J Am Soc Echocardiogr 2000;13:599-607.

15 Schwammenthal E, Popescu B, Popescu A, et al. Noninvasive assessment of left ventricular end-diastolic pressure by the response of the transmitral A-wave velocity to a standardized Valsalva maneuver. Am J Cardiol 2000;86:169-74.

16 Dumesnil JG, Gaudreault G, Honos GN, et al. Use of Valsalva maneuver to unmask left ventricular diastolic function abnormalities by Doppler echocardiography in patients with coronary artery disease or systemic hypertension. Am J Cardiol 1991;68:515-9.

17 Hurrell DG, Nishimura RA, Ilstrup DM, et al. Utility of preload alteration in assessment of left ventricular filling pressure by Doppler echocardiography: a simultaneous catheterization and Doppler echocardiographic study. J Am Coll Cardiol 1997;30:459-67

18 Keren G, Sherez J, Megidish R, et al. Pulmonary venous flow pattern--its relationship to cardiac dynamics. A pulsed Doppler echocardiographic study. Circulation 1985;71:1105-12.

19 Nishimura RA, Abel MD, Hatle LK, et al. Relation of pulmonary vein to mitral flow velocities by transesophageal Doppler echocardiography. Effect of different loading conditions. Circulation 1990;81:1488-97.

20 Kuecherer HF, Kusumoto F, Muhiudeen IA, et al. Pulmonary venous flow patterns by transesophageal pulsed Doppler echocardiography: relation to parameters of left ventricular systolic and diastolic function. Am Heart $J$ 1991; 122:1683-93.

21 Appleton CP, Galloway JM, Gonzalez MS, et al. Estimation of left ventricular filling pressures using two-dimensional and Doppler echocardiography in adult patients with cardiac disease. Additional value of analyzing left atrial size, left atrial ejection fraction and the difference in duration of pulmonary venous and mitral flow velocity at atrial contraction. J Am Coll Cardiol 1993;22:1972-82.

22 Rossvoll O, Hatle LK. Pulmonary venous flow velocities recorded by transthoracic Doppler ultrasound: relation to left ventricular diastolic pressures. J Am Coll Cardiol 1993:21:1687-96.

23 Brunazzi MC, Chirillo F, Pasqualini $M$, et al. Estimation of left ventricular diastolic pressures from precordial pulsed-Doppler analysis of pulmonary venous and mitral flow. Am Heart J 1994; 128:293-300.

24 Dini F, Michelassi C, Micheli G, et al. Prognostic value of pulmonary venous flow Doppler signal in left ventricular dysfunction: contribution of the difference in duration of pulmonary venous and mitral flow at atrial contraction. J Am Coll Cardiol 2000;36:1295-302.

25 Donovan CL, Armstrong WF, Bach DS. Quantitative Doppler tissue imaging of the left ventricular myocardium: validation in normal subjects. Am Heart J 1995; 130:100-4

26 Bach DS. Quantitative Doppler tissue imaging as a correlate of left ventricular contractility. Int J Cardiac Imaging 1996;12:191-5.

27 Garcia MJ, Rodriguez L, Ares M, et al. Myocardial wall velocity assessment by pulsed Doppler tissue imaging: characteristic findings in normal subjects. Am Heart J 1996;132:648-56.

28 Gulati VK, Katz WE, Follansbee WP, et al. Mitral annular descent velocity by tissue Doppler echocardiography as an index of global left ventricular function. Am J Cardiol 1996;77:979-84.

29 Rodriguez L, Garcia M, Ares $M$, et al. Assessment of mitral annular dynamics during diastole by Doppler tissue imaging: comparison with mitral Doppler inflow in subjects without heart disease and in patients with left ventricular hypertrophy. Am Heart J 1996;131:982-7.

30 Sohn DW, Chai IH, Lee DJ, et al. Assessment of mitral annulus velocity by Doppler tissue imaging in the evaluation of left ventricular diastolic function. J Am Coll Cardiol 1997;30:474-80.

31 Nagueh S, Middleton K, Koplen H, et al. Doppler tissue imaging: a noninvasive technique for evaluation of left ventricular relaxation and estimation of filling pressures. J Am Coll Cardiol 1997;30:1527-33.

32 Nagueh SF, Mikati I, Kopelen HA, et al. Doppler estimation of left ventricular filling pressure in sinus tachycardia. A new application of tissue doppler imaging. Circulation 1998;98:1644-50.

33 Lisauskas J, Singh J, Courtois M, et al. The relation of the peak Doppler $\mathrm{E}$-wave to peak mitral annulus velocity ratio to diastolic function. Ultrasound Med Biol 2001;27:499-507.

34 Thomas JD, Garcia MJ, Greenberg NL. Application of color Doppler M-mode echocardiography in the assessment of ventricular diastolic function: potential for quantitative analysis. Heart Vessels 1997;(suppl 12):135-7.

35 Kitabatake A. Propagation velocity of left ventricular filling flow measured by color M-mode Doppler echocardiography. J Am Coll Cardiol 1998;31:1445-6.

36 Garcia M, Smedira N, Greenberg N, et al. Color M-mode Doppler flow propagation velocity is a preload insensitive index of left ventricular relaxation: animal and human validation. J Am Coll Cardiol 1999:35:201-8.

37 Moller J, Poulsen S, Sondergaard E, et al. Preload dependence of color $\mathrm{M}$-mode Doppler flow propagation velocity in controls and in patients with left ventricular dysfunction. J Am Soc Echocardiogr 2000;13:902-9. 